How to reference this article Besutti, P. (2019). Storie di emancipazione: Virginia Ramponi Andreini (1583-1631) dal suocero al marito. Italica Wratislaviensia, 10(2), 65-83.

DOI: http://dx.doi.org/10.15804/IW.2019.10.1.17

Paola Besutti

Università degli Studi di Teramo

pbesutti@unite.it

ORCID: 0000-0002-5346-973X

\title{
STORIE DI EMANCIPAZIONE: VIRGINIA RAMPONI ANDREINI (1583-1631) DAL SUOCERO AL MARITO
}

\section{EMANCIPATION STORIES: VIRGINIA RAMPONI ANDREINI (1583-1631) FROM HER FATHER-IN-LAW TO HER HUSBAND}

\begin{abstract}
Numerous notarial deeds are kept in the State Archive of Mantua, involving some of the most famous comedians of the $17^{\text {th }}$ century. Piermaria Cecchini (Frittellino); Francesco Andreini and his sons Domenico and Giacinto; Giovan Battista (Lelio) with his son Pietro Enrico; Tristano Martinelli; and others used notaries for wills, sales contracts, debt reduction, inventories of assets, and dowries. Some of these documents also involve the women of the Andreini family: Lavinia (sister Fulvia), Caterina (perhaps sister Clarastella), Virginia Ramponi Andreini (Florinda). In particular, an emancipation deed (1620) indirectly affected Virginia (Genoa, 1583? - before 17 November 1631), the first wife of Giovan Battista Andreini.

On the basis of document analysis, two perspectives are explored: Virginia's peculiar contribution to the activities of the family, also from an economic point of view; and the status of women with regards to emancipation, which must be understood from a legal point of view that was patriarchal and defensive of property. The article thus becomes an opportunity to reflect, starting from Virginia but gazing even beyond, on the condition of women who were active in the theatrical and musical world between jurisprudence, artistic professions, economic heritage, and daily life. In conclusion, through some selected examples (the concerto delle dame of Ferrara, Adriana Basile, Margherita Salicola, Antonia Merighi), the theme of emancipation throughout the $17^{\text {th }}$ century is analysed, a period when women singers successfully populated the new operatic market. As the law remained unchanged, these women experimented with different strategies to protect their own person and assets.
\end{abstract}

Keywords: actress, female singers, opera, emancipation, law 
Jell'Archivio di Stato di Mantova sono conservati numerosi atti no1 tarili, che coinvolgono alcuni fra i più famosi comici del Seicento ${ }^{1}$. Piermaria Cecchini (Frittellino), Francesco Andreini e i suoi figli Domenico e Giacinto, Giovan Battista (Lelio) con suo figlio Pietro Enrico, Tristano Martinelli e altri, ricorsero ai notai per i loro testamenti, contratti d'acquisto e vendita, liberazione da crediti, inventari di beni e doti ${ }^{2}$. Alcuni di quegli atti, in parte noti ${ }^{3}$, interessano anche i membri femminili della famiglia Andreini: Lavinia (suor Fulvia), Caterina (forse suor Clarastella), Virginia Ramponi Andreini (Florinda).

Di peculiare interesse, un atto di emancipazione (1620) toccò indirettamente Virginia (Genova, 1583-?, ante 17 novembre 1631), prima moglie di Giovan Battista. Il documento diviene occasione per riflettere, partendo da Virginia stessa ma spingendo poi lo sguardo su tutto il Seicento, sulla condizione delle donne attive nel mondo teatrale e musicale, e sul concetto giuridico di emancipazione femminile fra giurisprudenza, professioni artistiche, patrimoni economici e vita quotidiana.

\section{1. "PER LA COMEDIA DI ARIANA"}

Il 31 gennaio 1620 Francesco Andreini e suo figlio Giovan Battista ricorsero a un notaio per rogare l'atto di emancipazione di Giovan Battista, allora quarantaquattrenne, dal padre settantaduenne ${ }^{4}$. In quel periodo la patria potestà non decadeva con il raggiungimento della maggiore età dei figli, fissata orientativamente a venticinque anni: il figlio raggiungeva infatti la piena indipendenza giuridica solo alla morte del padre ${ }^{5}$. Vivente il genitore era possibile anticipare l'emancipazione con un atto

1 ASMn, Decreti; ASMn, Notarile. I documenti sono stati in parte resi noti in Besutti, 1995 [1998], pp. 227-276.

2 Per un sintetico elenco degli atti si rinvia a Besutti, 1995 [1998], p. 229.

3 Un elenco di atti notarili è riportato in Burattelli, Landolfi, Zinanni, 1993, vol. 1, pp. 81, 85, 96; cfr. anche Ferrone, 1993, pp. 312-313.

4 ASMn, Notarile, notaio Giulio Cesare Pallini, 31 gennaio 1620; documento reso noto in Besutti, 1995 [1998].

5 Cfr. Pertile, 1894, vol. 3, pp. 381-86 e Enciclopedia del Diritto, vol. 14, pp. 807-828 . 
notarile, come anche ai nostri giorni è lecito fare nei confronti dei minorenni. L'emancipatio era possibile solo col consenso di entrambe le parti. Tale norma coinvolgeva a livello secondario anche le componenti femminili della famiglia che, in una struttura patriarcale e difensiva dei patrimoni, seguivano la linea maschile via via prevalente, ovvero paterna, matrimoniale o parentale.

Il citato atto di emancipazione mette in evidenza le molteplici attività di Giovan Battista, comico e scrittore, enumerando e ponderando in un allegato in lingua italiana $i$ doni da lui ricevuti, ma sino a quel momento confluiti nel patrimonio paterno (Besutti, 1995 [1998], pp. 272 -274). La scrittura si chiude con la ratifica dell'emancipazione, ossia con lo scioglimento del figlio dal vincolo della patria potestà («a nexu patrie potestatis»), che include anche la motivazione: più facile conduzione degli affari futuri («facilior ipsi sit in posterum negotiatio»). La maggiore autonomia professionale, resa necessaria anche dai viaggi fuori d'Italia ${ }^{6}$, è ribadita poco oltre ove la scrittura afferma che da quel momento Giovan Battista «tamquam homo liber» potrà: «testari, codicillari, vendere, aquirere, pacisci, in Judicio sistere, contrahere se et bona sua obligare, ac deinceps omnia alia et singula dicere, facere et exercere quae pater familias, et homo sui juris potest» (Ibidem, p. 230) ${ }^{7}$.

Di rilevante interesse risulta il citato elenco di donativi, attestante i guadagni di Giovan Battista e implicitamente di sua moglie Virginia che, per legge, era sottoposta alla potestà del marito e dunque, transitivamente, a quella del suocero, almeno sino al momento dell'emancipazione del marito dal proprio padre.

I regali ricevuti, consistenti prevalentemente in gioielli e oggetti preziosi, vengono non solo sommariamente descritti e valutati in scudi, ma anche ascritti al donatore e al motivo dell'elargizione. Tale forma di remunerazione in solido ovvero sotto forma di collane, abiti, complementi di abbigliamento o altro, era talmente diffusa da configurarsi

\footnotetext{
6 Sui viaggi transalpini cfr. anche Besutti, 2005.

7 Traduzione: «far testamento, modificarlo, vendere, acquistare, concludere contratti, comparire in Giudizio, aver relazioni d'affari, impegnare i propri beni, e inoltre dire, fare e condurre tutto quanto è nei diritti del capo famiglia e dell'uomo».
} 
come fenomeno sistemico, che ha di recente attratto l'attenzione degli storici dell'economia (Rollandi, Romani, 2018).

Dall'elenco emergono titoli di opere pubblicate da Giovan Battista e dedicate a qualche notabile personaggio, ma anche titoli di testi recitati, esplicitamente menzionati o almeno congetturalmente identificabili. Al di là dei singoli eventi, è utile sottolineare che i dati economici menzionati contribuiscono a chiarire le diverse fonti e forme di reddito delle quali potesse disporre un'affermata coppia di comici e quali vantaggi materiali, oltre che di prestigio, producesse l'attività scrittoria, la mercatura teatrale e, nel caso di Virginia, la sempre più quotata pratica del canto all'interno degli emergenti generi delle azioni teatrali con musica, dell'opera in musica e delle forme rappresentative da camera.

Tra le righe dell'atto di emancipatio traluce la difficoltà nella quantificazione dei beni derivanti da attività spesso indiscernibili tra loro. Se infatti la valutazione dei doni ricevuti per la dedica di opere era agevole, in quanto derivante da fatti certi, ben più arduo doveva essere il computo dei proventi derivanti dalla pratica quotidiana del teatro, che infatti viene descritta sommariamente in una formula cumulativa, significativamente priva di valutazione in denaro: «Senza tanti altri regali di gentilomini gentildone et altri particolari cioè il guadagno ordinario della comedia».

Nello stesso allegato figurano, senza specifiche distinzioni, doni ricevuti dai diversi membri della famiglia attoriale come ricompensa per le attività teatrali ordinarie: 1000 scudi in regali d'oro dai sovrani di Francia in occasione del viaggio transalpino del 1613-1614; 72 pezzi d'oro (100 scudi) dal cardinale Luigi Capponi, legato di Bologna (1614-1619), forse per le recite dei Fedeli nell'autunno 1618; "gioielli, pendenti e fiori» (Besutti, 1995 [1998], p. 273) del valore di 250 ducati dal duca Vincenzo I Gonzaga in occasione di non meglio definite feste di Natale e dell'Ascensione; un vestito del valore di circa 210 scudi da Carlo di Lorena duca di Guisa, forse per le recite in Francia del 1613$-1614^{8}$; una riffa di un diamante a Genova, forse per recite tenute nel

8 L'anno successivo all'atto notarile, Giovan Battista Andreini dedicò al duca La campanazza (Andreini, 1621), alcuni esemplari della quale esibiscono tale dedica. 
maggio $1611^{9}$; una licenza per il territorio di Mantova dal duca Ferdinando Gonzaga.

È probabile che parte dei beni cumulativamente elencati fossero stati guadagnati anche da Virginia. Almeno in un caso c'è al riguardo qualche indizio esplicito: è documentato infatti che le recite bolognesi dei comici Fedeli per il cardinale Capponi (autunno 1618) furono favorite dalle raccomandazioni di don Giovanni de' Medici e da una sosta a Bologna degli Andreini nell'ottobre 1617, che aveva fruttato "doni" proprio a Florinda (Burattelli, Landolfi, Zinanni, 1993, vol. 1, p. 482).

Alcune occasioni rappresentative, anteriori al gennaio 1620, vengono poi registrate con maggior dovizia di particolari forse in virtù della loro eccezionalità; nella maggior parte dei casi si riferiscono al periodo dei duchi Gonzaga Vincenzo I (1562-1612), Francesco IV (1586-1612) e Ferdinando (1587-1626):

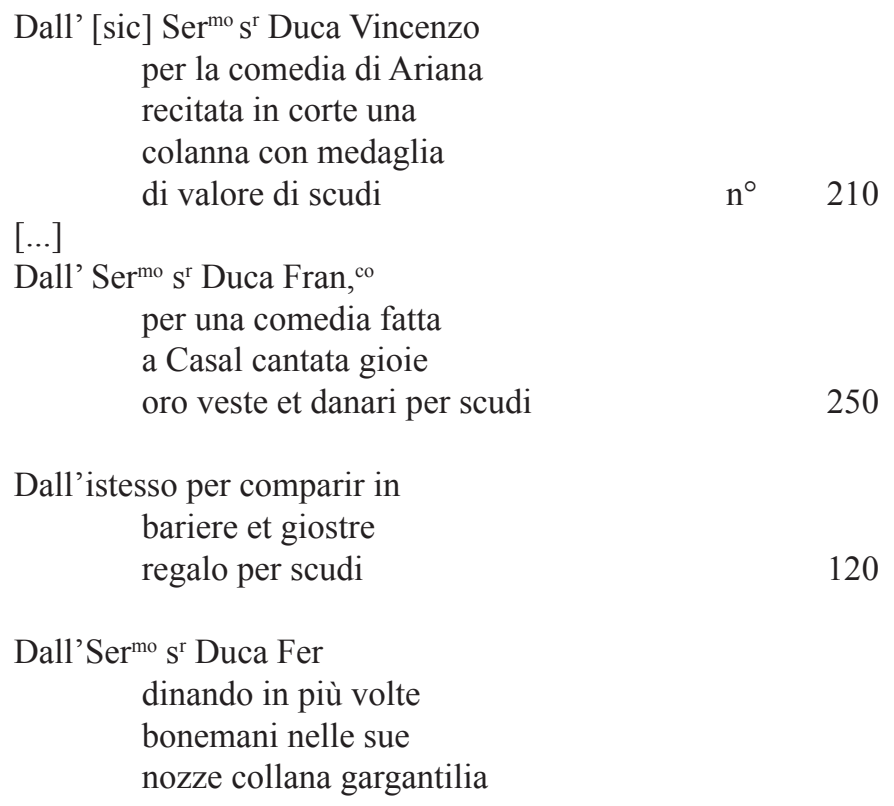

Dall'Sermo ${ }^{\mathrm{r}}$ Duca Fer

dinando in più volte

bonemani nelle sue

nozze collana gargantilia

9 Gli Andreini furono a Genova probabilmente nel maggio 1611, cfr. Burattelli, Landolfi, Zinanni, 1993, vol. 1, pp. 372-73; vol. 2, p. 17. 
in tutto alla somma di

scudi

1800

(Besutti, 1995 [1998], pp. 272-273)

Nella prima voce viene menzionata la «comedia di Ariana» verosimilmente identificabile con quella rappresentata nelle ben note feste mantovane del 1608 per le nozze di Francesco con l'infanta Margherita di Savoia. Le feste, che coinvolsero gli Andreini (i Fedeli) nella recita dell'Idropica (Teatro grande, 2 giugno) di Battista Guarini con fastosi intermedi, sancirono soprattutto il trionfo di Virginia nei ruoli cantati di Arianna nell'omonima tragedia per musica di Ottavio Rinuccini e Claudio Monteverdi (Teatro temporaneo, 28 maggio), e di Ingrata nel Ballo delle ingrate composto dagli stessi (Teatro grande, 4 giugno) ${ }^{10}$.

Federico Follino nella propria celeberrima cronaca così descrisse la performance di Virginia in Arianna:

[...] e venendo rappresentata sì da uomini, come da donne nell'arte del canto eccellentissime, in ogni sua parte riuscì più che mirabile, nel lamento che fece Arianna sovra lo scoglio, abbandonata da Teseo, il quale fu rappresentato con tanto affetto, e con sì pietosi modi, che non si trovò ascoltante alcuno, che non s'intenerisse, nè fu pur una Dama, che non versasse qualche lagrimetta al suo bel pianto. $(1608$, p. 29$)$

Il lamento, unica parte dell'opera a oggi reperibile, divenne un'icona del potere incantatorio della musica unita alla parola e al gesto; c'è chi ha ipotizzato che il lamento sia stato aggiunto al dramma in fase di elaborazione proprio su suggerimento di Virginia (Carter, 1999), magari dopo la prova che si tenne nel febbraio 1608 nella Sala degli Specchi del palazzo Ducale (Besutti, 1999b), a pochi giorni dalla morte per vaiolo della cantatrice Caterina Martinelli (1590 ca.-1608), che avrebbe dovuto impersonare Arianna. Il minimo margine di dubbio sull'identificazione della rappresentazione con quella del maggio 1608 discende

10 Le feste mantovane del 1608 furono descritte in: Follino, 1608 (facsimile in Gallico, 2004); Bertazzolo, 1608. Della bibliografia moderna si ricordino almeno: Solerti, 1903, pp. 73-103; Fabbri, 1985, pp. 124-148; Ferrone, 1993, p. 132, nota 66; Besutti, 1999c; 2009, pp. 76-77. 
da un'antica ipotesi, mai confermata, di un'altra esecuzione "in corte" nel 1612, anzi entro il 18 febbraio 1612, data di morte di Vincenzo I, dispensatore dell'elargizione (Solerti, 1903, vol. 1, pp. 116-117, nota 5; Parisi, 1989, p. 273, nota 87$)^{11}$. Si ricordi che anche una successiva recita di Arianna, progettata (1620) dal duca Ferdinando Gonzaga, non ebbe luogo (Besutti, 2005b; 2013).

È assai verosimile che il dono collegato nel documento all'Arianna sia da ascrivere proprio alle prestazioni canore e performative di Virginia per almeno due motivi: anzitutto poiché l'apprezzamento suscitato dalla sua interpretazione fu tale da giustificare il generoso regalo, in secondo luogo per il fatto che, al di là dell'uso della parola "recitata", non sussistono al momento elementi per supportare l'ipotesi di un'eventuale partecipazione non cantata di Giovan Battista nella rappresentazione della tragedia per musica stessa.

Ancora a Virginia sono con buona probabilità da attribuire i doni ricevuti per la commedia «fatta a Casal cantata» (Besutti, 1995 [1998], p. 273), quasi certamente identificabile con la favola $I l$ rapimento di Proserpina di Ercole Marliani, musicata da Giulio Cesare Monteverdi (Casale, 29 aprile 1611) ${ }^{12}$. Già sposato con Margherita di Savoia, in quel periodo Francesco Gonzaga non era ancora duca, ma principe ereditario e governatore di Casale, che dotò, quasi come seconda corte autonoma rispetto a quella paterna di Mantova, di proprie maestranze musicali, guidate dal maestro di cappella Giulio Cesare Monteverdi $\left(1573\right.$ - 1630) ${ }^{13}$. Alla recita di Il rapimento di Proserpina contribuirono i migliori vir-

11 Fabbri (1985, p. 202) ipotizza anche una ripresa fiorentina nel 1614. Nella corte di Mantova venne ipotizzata, ma non realizzata una ripresa nel 1620 (Besutti, 2005b; 2013). Non si ha notizia circa un'eventuale rappresentazione recitata non in canto da parte di Virginia del ruolo di Arianna, documentata invece per altre comiche professioniste, come Celia Malloni e Marina Dorotea Antonazzoni, che lo interpretò, tra l'altro, a Firenze (1616), cfr. Burattelli, Landolfi, Zinanni, 1993, vol. 1, pp. 458, 502; Ferrone, 1993, p. 268, nota 75; Fabbri, 1985, p. 383, nota 41.

12 Breve descrittione delle feste Fatte dal Serenissimo Sig. Principe di Mantova, (1611); trascrizione parziale in Solerti, 1903, vol. 1, pp. 157-161. Si noti la coincidenza editoriale con Andreini, 1611.

13 Sulle diverse cappelle contemporaneamente attive nella corte dei Gonzaga cfr. Carter, 2012 [2016]; Besutti, 2017; 2018 b. 
tuosi del momento: i tenori Francesco Rasi e Francesco Campagnolo e, appunto, Virginia nel ruolo di Cerere. La sua interpretazione venne così descritta: «Venne la famosa Sig. Florinda, Idea del bel dire, Gloria de' Comici, Pompa de' Teatri et così efficace spiegatrice degli affetti dell' animo che col pietoso canto mosse altri al pianto» (Breve descrittione delle feste Fatte dal Serenissimo Sig. Principe di Mantova, 1611) ${ }^{14}$. Tale descrizione, che riprende quasi letteralmente quella formulata da Follino nel 1608, consacra il potenziale emozionale del nuovo genere operistico e soprattutto la carismatica capacità di Virginia di muovere gli affetti più profondi, grazie a un "non so che" interpretativo che le era proprio $^{15}$.

L'annotazione relativa ai doni ricevuti «per comparir in bariere et giostre» (Besutti, 1995 [1998], p. 273) non offre per il momento elementi di collegamento con Virginia, anche se non è da escludere la sua comparsa nei tornei del periodo, magari nel prologo, spesso accompagnato da carri e apparati arricchiti dal canto e dai musici (Besutti, 1999).

La quarta annotazione notarile enumera i regali ricevuti da Giovan Battista e quasi certamente da Virginia in occasione delle nozze del duca Ferdinando Gonzaga con Caterina de' Medici, celebrate a Firenze 1'ultimo giorno di carnevale ( 7 febbraio 1617) e festeggiate a Mantova dall' 8 marzo successivo. A Firenze, forse per il coincidente inizio della quaresima, le manifestazioni di allegrezza furono limitate a due soli eventi teatrali (Solerti, 1905, p. 124): la «veglia reale» La liberazione di Tirreno e d'Arnea, autori del sangue toscano (6 febbraio 1617) ${ }^{16}$, e una «festa spirituale» alla compagnia di S. Marco in via di San Gallo (9 febbraio 1617) ${ }^{17}$. A Mantova l'evento culminante fu la fastosa ripresa (Solerti,

\footnotetext{
14 Citata anche in Cohen, 1986, p. 424.

15 Sul concetto di "non so che" in musica cfr. Besutti, 2003; Eadem, in stampa.

${ }^{16}$ La liberazione di Tirreno e d'Arnea, autori del sangue toscano. Veglia reale, testo di Andrea Salvadori, musiche di Marco da Gagliano, coreografie di Agnolo Ricci, controversa la partecipazione di Jacopo Peri; cfr. Weaver, Wright Weaver, 1978, pp. 100-101; Kirkendale, 1993, pp. 225, 605, 611.

17 Cfr. Solerti, 1905, p. 124.
} 
1905, p. 124) di La Galatea del fiorentino Santi Orlandi, maestro della musica del duca Ferdinando, su versi di Gabriello Chiabrera ${ }^{18}$.

Nessun documento ha sinora confermato una realizzazione in quei giorni a Mantova di La Maddalena, sacra rappresentazione di Giovan Battista Andreini ${ }^{19}$. La coeva stampa delle musiche di Monteverdi, Effrem, Guivizzani e S. Rossi per La Maddalena ${ }^{20}$ nonché la presenza, in calce alla nuova edizione del testo, di indicazioni sceniche ${ }^{21}$, paiono tuttavia vincolare la revisione ed edizione del 1617 a un'occasione spettacolare, collegata ragionevolmente con le feste nuziali. Ammesso che la recita con musiche di La Maddalena sia realmente avvenuta in coincidenza con le nozze, la rappresentazione, diversamente da quanto sinora ipotizzato, avrebbe potuto aver avuto luogo a Firenze, dove è appunto documentata una «festa spirituale» e dove era stata stampata una delle sue prime edizioni (Andreini G.B., 1612).

Sebbene l'allegato all'emancipatio confermi l'effettiva partecipazione degli Andreini alle feste nuziali del 1617, non è tuttora possibile stabilire con certezza quali prestazioni avessero motivato l'esorbitante elargizione di doni per il valore di ben 1800 scudi. Virginia avrebbe potuto cantare nella Galatea e in La Maddalena, mentre Giovan Battista e gli altri membri della compagnia sarebbero potuti intervenire negli intermedi a La Galatea stessa; di questi non resta traccia, ma furono allora giudicati «dei più belli che si facciano» (Portioli, 1882, p. 15) ) $^{22}$.

L'atto di emancipatio lascia dunque trasparire lo specifico contributo di Virginia, attrice-cantante, all'economia della famiglia. Il fatto che,

18 Sulle nozze e sulla rappresentazione: Portioli, 1882; Ademollo, 1888, p. 234; Solerti, 1905, p. 125; Parisi, 1989, pp. 305-308; Besutti, 1996, pp. 59-62.

19 L'ipotesi fu formulata da Canal, 1881, p. 117, sostenuta da Bevilacqua, 1894, pp. 98-101 e ripresa da: Fabbri, 1985, p. 211; Harrán, 1987, p. 102; Carandini, 1995, p. 447; Parisi, 1989, p. 307.

20 Musiche de alcuni eccellentissimi musici, 1617. Edizione moderna: Harrán, 1995, vol. 8, XLVII-LI, pp. 48-67.

21 «Avisi per alcuni effetti nell'opera recitativa» (Andreini, 1617). Il soggetto di La Maddalena era già stato precedentemente trattato da "Lelio" in forma di poema: Andreini, 1610, 1612, 1628 (con varianti); il testo del 1617 fu riedito (Idem, 1620) e ripreso con varianti in Idem, 1652.

22 Si segnala inoltre la pubblicazione in coincidenza con le nozze di Andreini, $1617 \mathrm{~b}$. 
come del resto ci si attenderebbe, quello stesso atto non la menzioni mai esplicitamente rende palese, da altra prospettiva, l'invisibilità delle donne "professioniste" nel mondo della musica e del teatro, almeno sotto il profilo giuridico. Il che avveniva anche per colei che fu tanto celebre da divenire 1'emblema di un gruppo di comici talvolta menzionato come la «compagnia della Florinda» (Carandini, Mariti, 2003, p. 252).

Tale legislazione, che di fatto privava le donne della possibilità di ereditare in via diretta beni e proprietà, non impediva loro del tutto di muoversi in ambito economico, ma a un livello spicciolo e quotidiano oppure ricorrendo a strategie utili a aggirare gli ostacoli giuridici. $\mathrm{Si}$ ricordi, per esempio, che Barbara Mangini, sorella del comico Andrea Mangini (Adriano), ricevette una serie di pagamenti proprio da Virginia fra il 1622 e il $1625^{23}$. Molte testimonianze confermano del resto come fossero proprio le donne a gestire parte degli affari economici delle compagnie. Benché dunque le attrici e, come vedremo tra poco, le cantatrici andassero acquisendo dalla fine del Cinquecento un potere economico crescente, la distanza giuridica e sociale dagli uomini restava profonda.

In un momento in cui l'orizzonte professionale femminile era ancora piuttosto limitato, i proventi del lavoro artistico di Virginia erano confluiti dunque nel patrimonio del suocero per passare, dopo la citata emancipatio, in quello del marito. Tale vincolo contribuisce a spiegare anche perché molte donne, e Virginia fra esse, accettassero di mantenere il vincolo matrimoniale anche in situazioni imbarazzanti quali la coesistenza con amanti "ufficiali” del marito, come fu Virginia Rotari (Lidia) per Giovan Battista.

Nei decenni a venire le occasioni di lavoro artistico per le donne si moltiplicarono, ma a tale accelerazione non corrispose un aggiornamento delle norme giuridiche.

23 ASMn, Notarile, notaio Giulio Cesare Pallini, 26 ottobre 1622 e 14 luglio 1625; l'atto del 1622 è menzionato in Burattelli, Landolfi, Zinanni, 1993, vol. 1, p. 116. 


\section{STRATEGIE FEMMINILI DOPO VIRGINIA}

Il trionfo canoro di Virginia nel Seicento traccia un segno di discontinuità artistica e professionale nella storia del canto al femminile e della nascente opera in musica. La sua condizione di attrice-cantante diverge da quella delle cantatrici "pure" attive nelle stesse corti padane di Ferrara e Mantova. Ella poteva infatti calcare le scene impersonando da professionista ruoli femminili, solitamente preclusi alle donne: si ricordi che nell'Orfeo di Alessandro Striggio il giovane e Claudio Monteverdi, rappresentato in ambito accademico (24 febbraio 1607), tutti gli interpreti furono uomini.

Pochi anni prima l'onorabilità delle famosissime cantatrici e strumentiste della corte di Ferrara fu difesa con la qualifica di dame di compagnia della duchessa che marcava la differenza dal mondo dei teatri (Besutti, 2003), il che tuttavia nel tempo non le salvaguardò dai pericoli. Le insidie sociali che circondavano le "professioniste" erano note alle donne di rango che ricoprivano, per diritto di sangue, posizioni politicamente e socialmente apicali. La duchessa Margherita Gonzaga d'Este (1564-1618) stese una metaforica rete di protezione attorno al concerto delle sue «dame principalissime» (Durante, Martellotti, 1989) di Ferrara, che non lo preservò da un triste epilogo: quando ella, divenuta vedova, ritornò a Mantova per la devoluzione del ducato di Ferrara, il concerto si disperse e Anna Guarini fu addirittura assassinata dal marito ${ }^{24}$.

Margherita Gonzaga d'Este, donna forte e volitiva, nella sua nuova posizione di duchessa vedova senza ducato non cessò di occuparsi della condizione femminile, favorendo in patria la nascita di un convento di Orsoline. L'ordine, fondato come compagnia laica all'inizio del Cinquecento da Angela Merici, nel 1584 fu accettato dal vescovo Paolo Leoni a Ferrara, dove Margherita lo conobbe. Tale realtà offriva alle donne un'alternativa rispetto alla stringente scelta fra matrimonio o convento di clausura poiché consentiva alle donne l'elevazione culturale e, nel caso della regola mantovana, il mantenimento dei propri beni, altrimenti non consentito. Il convento divenne una vera e propria corte monastica

24 Sul concerto delle dame di Ferrara cfr. Durante, Martellotti, 1989; 2010. 
e un collegium nobilium femminile nel quale, protette, vissero e si formarono non solo le principesse di casa Gonzaga e le giovani dell'aristocrazia territoriale, ma anche artiste come la pittrice Giustina Fetti (suor Lucrina) che vi poté operare indisturbata ${ }^{25}$.

Altre coeve dive del canto, come Adriana Basile (1580-1642), protessero la propria figura circondandosi dei più fidati componenti maschili della famiglia, che garantirono loro l'onorabilità sociale e la difesa patrimoniale ${ }^{26}$.

Le opere rappresentate nei teatri pubblici dagli anni Trenta del Seicento in avanti in numero vertiginosamente crescente, sempre più spesso esplicitano i nomi delle cantanti donne che, accanto ai castrati, interpretavano diversi ruoli con grandi riconoscimenti economici. Il fatto tuttavia che la loro condizione giuridica fosse immutata le indusse a ingegnarsi per trovare nuove strategie di salvaguardia.

La dichiarazione del legame di una cantante con un nobile o con un regnante, manifestata dalla formula ricorrente «virtuosa del» (del duca o del principe o di altra personalità di rango) è un segno di questo fenomeno. La contabilità riservata dagli uffici di corte alle numerose cantatrici dell'ultimo duca di Mantova, Ferdinando Carlo Gonzaga (1652-1708), attesta da un lato la mobilità del nuovo mercato operistico, e da un altro lato la necessità della tutela nobiliare e maschile. Le carte elencano meticolosamente $\mathrm{i}$ compensi in denaro e in beni di consumo riconosciuti a ciascuna cantatrice e dettagliano il nucleo familiare delle stesse, che molto spesso, in assenza di un padre, o di un marito o di un fratello contemplava un non meglio definito "zio" ovvero una figura maschile che poteva compiere atti ufficiali preclusi alle donne, quale l'acquisto di immobili (Besutti, 1989; 1997).

La protezione ducale, che si esplicava anche nel rilascio di patenti e passaporti, necessari per muoversi fra $i$ vari stati che componevano l'Italia di allora, sanciva anche una forma di possesso nei confronti delle artiste, il che in nuovo mercato operistico creò spesso conflitti fra dipendenza e libertà professionale. Ben noto è il caso della cantante Margherita

25 Su Margherita Gonzaga cfr. Besutti, 2018.

$26 \mathrm{Su}$ Adriana Basile cfr., anche per altri riferimenti bibliografici, Megale, 2016. 
Salicola (1670-1717) ${ }^{27}$ : dopo aver rifiutato (1683) il matrimonio fra pari con il tenore Giovanni Buzzoleni che, oltre a una ricca dote, chiedeva il suo ritiro dalle scene ${ }^{28}$, divenne "virtuosa" del duca Ferdinando Carlo Gonzaga (2 gennaio 1685); Giovanni Giorgio III, principe elettore di Sassonia, sentendola cantare a Venezia ne rimase folgorato e le propose di trasferirsi a Dresda, offrendole 10.000 lire annue, più altre 5000 per la famiglia rimasta in Italia, e inscenando un finto rapimento che scatenò un clamoroso caso diplomatico. Il duca di Mantova, dopo aver fatto segregare tutta la famiglia della giovane giunse a sfidare l'elettore di Sassonia; grazie all'intervento del principe elettore di Baviera i due contendenti si scusarono e Margherita poté rimanere a Dresda (Ademollo, 1888 , p. 532), dove divenne prima cantatrice, amante ufficiale dell'elettore, nonché madre di un figlio che egli riconobbe ${ }^{29}$. Dopo essere passata sotto la protezione dell'Imperatore Leopoldo I, Margherita tornò in Italia dove sposò il modenese Marc'Antonio Suini che, a differenza di Buzzoleni, non le impedì di esercitare la sua professione, protetta anche dal duca di Parma e da quello di Modena. Sfruttata sin da piccola dalla famiglia d'origine, Margherita riuscì dunque a destreggiarsi fra diverse forme di protezione maschile.

In modo meno spregiudicato, altre cantanti riuscirono a creare sodalizi con colleghi maschi, spesso castrati. Si ricordino, tra gli altri, i casi del castrato Nicola Grimaldi che ebbe a cuore le sorti della collega Marianna Benti Bulgarelli (la Romanina), o del Senesino che si prodigò per Margherita Durastanti e per Maria Maddalena Frigeri Salvai ${ }^{30}$.

Tra i casi recentemente documentati spicca quello del contralto bolognese Antonia Merighi (1690 ca.-1764 ca.), che creò un saldo sodalizio con il collega castrato Antonio Bernacchi ${ }^{31}$, il quale si preoccupava

27 Le vicende biografiche della Salicola sono recentemente state approfondite da Anzani, in stampa.

28 Su Buzzoleni cfr. Besutti, 1992; Monaldini, 2000, pp. 454, 456, 552, 573, 580.

29 Johann Georg Maximilian von Fürstenhoff; visse e morì (15 luglio 1753) a Dresda, dove divenne famoso architetto (Neue Deutsche Biographie, 1961, vol. 5, p. 699).

30 Documenti in Anzani, in stampa. Ringrazio l'autrice per avermi concesso il suo saggio in lettura.

31 Su Bernacchi e la Merighi cfr. Anzani, pp. 1917-18. 
di far avere alla collega buoni contratti, decidendo in sua vece quando il titolo di "virtuosa della gran principessa Violante di Toscana" non era garanzia sufficiente. I gruppi familiari dei due cantanti si aiutavano sottoscrivendo, tra l'altro, atti notarili che la cantante sola non avrebbe potuto gestire. Quando Angela acquistò a Bologna il nobile Palazzo Scarselli dovette ricorrere al consenso del padre, che confermò come l'acquisto si basasse su beni della figlia, usufruttuaria dell'immobile. Alla morte del padre, la tutela del patrimonio di Antonia sarebbe passata ad Antonio Gozzi, marito della sorella Daria. Per garantire i propri beni ella, non potendo sposare Bernacchi, al quale in quanto castrato era precluso il matrimonio, sposò il suo più vicino allievo Carlo Carlani, che risulta vivesse sotto lo stesso tetto del maestro. Tale passo consentì ad Antonia di difendere i propri averi sino alla successione testamentaria.

In antico regime nei riguardi di un uomo era difficile stabilire in modo univoco persino il limite della maggiore età, poiché era legato al tipo di ordinamento cui si era sottoposti, che poteva discendere dal diritto comune, o dal diritto della città e delle corporazioni, o dal diritto canonico, e poteva dunque variare a seconda dei diversi atti ai quali si doveva accedere, quali il matrimonio, la stipula di contratti, il diritto di testare. La legge tuttavia dava agli uomini gli strumenti per affrancarsi e per normare la propria autonomia.

Lo status delle donne era invece netto, e almeno sotto il profilo del diritto, senza vie di fuga. Esse infatti restavano sempre sotto la tutela maschile, passando con il matrimonio dalla potestà del padre a quella del marito o, in caso di mancato matrimonio, dal padre a quella del familiare maschio più anziano, anche se parente non prossimo. Tale sistema di fatto vincolava l'imprenditorialità femminile a un soggetto maschile di riferimento parentale (padre, marito, suocero, fratello, zio, cugino, cognato o altro) e, in mancanza del consenso o della tutela maschile, privava la donna del diritto di entrare in possesso dei beni stabili acquisiti con le proprie sostanze (Barbagli, 1984, pp. 189-203).

I casi di Virginia Andreini, Margherita Salicola e Antonia Merighi dimostrano che le donne, di fatto entrate a pieno titolo nel mondo delle professioni artistiche, si mantennero nella sfera della famiglia oppure si ingegnarono per trovare strategie di vita alternative, che consentissero 
loro di aggirare un diritto di emancipazione invariato rispetto alla logica patriarcale.

Lista delle abbreviazioni bibliografiche:

ASMn - Archivio di Stato di Mantova

\section{BIBLIOGRAFIA}

Ademollo, A. (1888). La bell'Adriana ed altre virtuose del suo tempo alla corte di Mantova. Città di Castello: Lapi.

Andreini, F. (1611). L'ingannata Proserpina. Venezia: Somasco.

Andreini, G.B. (1610). La Maddalena. Venezia: Somasco.

Andreini, G.B. (1612). La Maddalena. Firenze: Heredi di Cristofano Marescotti.

Andreini, G.B. (1617). La Maddalena, sacra rappresentazione. Mantova: Osanna.

Andreini, G.B. (1617b). Fama consolatrice nelle Reali Nozze de'Serenissimi Sposi Ferdinando Gonzaga e Caterina Medici nell'andata di S.A.S. a Fiorenza. Mantova: Osanna.

Andreini, G.B. (1620). La Maddalena. Milano: Malatesta.

Andreini, G.B. (1621). La Campanazza, commedia di Giovanni Rivani da Bologna, detto il Dottor Campanaccio da Budri. Parigi: Della Vigna.

Andreini, G.B. (1628). La Maddalena. Praga: Leva.

Andreini, G.B. (1652). La Maddalena lasciva e penitente, azzione drammatica, e divota in Milano rappresentata. Milano: Malatesta.

Anzani, V. (2017-2018). Antonio Bernacchi (1685-1756), virtuoso e maestro di canto bolognese (Ph.D. Thesis). Bologna: Università degli Studi di Bologna.

Anzani, V. (to appear). Patrimoni, matrimoni e tresche di virtuose, cantante, canterine e figlie da camera attraverso le lettere di supplica ai loro Serenissimi Padroni. In M. Mazzeo \& I. Yordanova (Eds.), Padron mio colendissimo...": Letters about Music and Stage in the $18^{\text {th }}$ Century. Queluz: Divino sospiro.

Barbagli, M. (1984). Sotto lo stesso tetto: mutamenti della famiglia in Italia dal XV al XX secolo. Bologna: il Mulino. 
Bertazzolo, G. (1608). Breve descrittione della battaglia navale, et del castello de fochi trionfali, fatti il dì 31 di Maggio 1608 sul lago di Mantova, nelle gloriosissime Nozze del Sereniss. Prencipe di Mantova, et di Monferrato, con la Serenissima Infanta D. Margherita di Savoia. Mantova: Heredi Francesco Osanna.

Besutti, P. (1989). La corte musicale di Ferdinando Carlo Gonzaga ultimo duca di Mantova. Musici, cantanti e teatro d'opera tra il 1665 e il 1707. Mantova: Arcari.

Besutti, P. (1992). Buzzoleni, Giovanni. In S. Sadie (Ed.), The New Grove Dictionary of Opera (vol. 1, p. 662). London: Macmillan.

Besutti, P. (1995). Da 'L'Arianna' a 'La Ferinda': Giovan Battista Andreini e la "comedia musicale all'improviso". Musica Disciplina, 49, 227-276.

Besutti, P. (1996). Domenico Fetti e la musica: vissuta, dipinta, rappresentata. In E.A. Safarik (Ed.), Domenico Fetti (pp. 59-62). Milano: Electa.

Besutti, P. (1997). La figura professionale del cantante d'opera: le virtuose di Ferdinando Carlo Gonzaga. Quaderni storici, 22/2, 409-433.

Besutti, P. (1999). Giostre, fuochi e naumachie a Mantova fra Cinque e Seicento. In P. Fabbri (Ed.), Musica in torneo nell'Italia del Seicento (pp. 3-32). Lucca: LIM.

Besutti, P. (1999b). The 'Sala degli Specchi' uncovered: Monteverdi, the Gonzagas and the Palazzo Ducale, Mantua. Early Music, 27/3, 451-464.

Besutti, P. (1999c). Il matrimonio dell'infanta Margherita: le feste a Mantova. In M. Masoero, S. Mamino \& C. Rosso (Eds.), Torino, Parigi, Madrid: politica e cultura nell'età di Carlo Emanuele I (pp. 491-506). Firenze: Olschki.

Besutti, P. (2003). 'Pasco gli occhi e gli orecchi': la rilevanza dell' 'actio' nella produzione e nella ricezione musicale tra Cinque e Seicento. In A. Pontremoli (Ed.), Il volto e gli affetti. Fisiognomica ed espressione nelle arti del Rinascimento (pp. 281-300). Firenze: Olschki.

Besutti, P. (2005). 'Cose all'italiana' e alla tedesca 'in materia di ricreatione': la circolazione di strumenti, strumentisti e balli fra Mantova e i territori dell'impero romano germanico (1500-1630). In U. Artioli \& C. Grazioli (Eds.), I Gonzaga e l'Impero. Itinerari dello spettacolo (pp. 239-272). Firenze: Le Lettere.

Besutti, P. (2005b). Variar 'le prime 7 stanze della luna': ritrovati versi di ballo per Jacopo Peri. Studi musicali, 34/2, 319-374.

Besutti, P. (2009). Federico Follino. Compendio delle sontuose [...] 1608. In C. Arnaldi di Balme \& F. Varallo (Eds.), Feste barocche. Cerimonie 
e spettacoli alla corte dei Savoia tra Cinque e Settecento (pp. 76-77). Milano: Silvana.

Besutti, P. (2013). The 1620s: the rebirth of 'Arianna'. Studi musicali, 2, 259-282 .

Besutti, P. (2017). Monteverdi's 'Daily Bread': The Economic Life of a Professional Musician. Early Music, 45/3 (August), 353-363.

Besutti, P. (2018). La musica e Margherita Gonzaga d'Este tra due corti e un convento. In C. Continisio (Ed.), Donne Gonzaga a corte. Reti istituzionali, pratiche culturali e affari di governo (pp. 445-461). Roma: Bulzoni.

Besutti, P. (2018b). Claudio Monteverdi cittadino mantovano. 'Tirsi e Clori', le feste del 1615 e il 'novo ordine generale'. Philomusica on-line, 17, $19-53$.

Besutti, P. (to appear). Il 'non so che' in Zarlino. In L. Zanoncelli (Ed.), 'Musico perfetto' Gioseffo Zarlino (1517-1590). Venezia: Fondazione Levi.

Bevilacqua E. (1894). Giambattista Andreini e la compagnia dei Fedeli. Giornale storico della letteratura italiana, 24, 82-165.

Bragato, A. (2016). Ramponi, Virginia, detta Florinda. In Dizionario Biografico degli Italiani, vol. 86. Roma: Istituto dell'Enciclopedia Italiana. Retrieved from http://www.treccani.it/enciclopedia/ramponi-virginiadetta-florinda_(Dizionario-Biografico)/

Breve descrittione delle feste Fatte dal Serenissimo Sig. Principe di Mantova Nel giorno natale della Serenissima Infanta Margherita. Et nella venuta delli Serenissimi Principe di Savoia Nella Città di Casale per veder detta signora, et il sig. principe prima della lor partita per Mantova (1611). Casale: Pantaleone Goffi.

Canal, P. (1977). Della musica in Mantova. Notizie tratte principalmente dall'Archivio Gonzaga. Bologna: Forni (Reprint, ed. originale: Mantova 1881).

Carandini, S., \& Mariti, L. (Eds.). (2003). Don Giovanni o L'estrema avventura del teatro. Roma: Bulzoni.

Carter, T. (1999). Lamenting Ariadne? Early music, 27/3, 395-405.

Carter, T. (2012). Monteverdi and some problems of biography. Journal of Seventeenth Century Music, 18/1. Retrieved from https://sscm-jscm.org/ jscm-issues/volume-18-no-1/.

Cohen, J. (1986). Giovan-Battista Andreini's dramas and the biginnings of opera. In M. Honegger \& P. Prevost (Eds.), La musique et le rite sacré et 
profane (vol. 2, pp. 423-432). Strasbourg: Association des Publications près les Universités de Strasbourg.

Durante, E., Martellotti A. (1989). Cronistoria del concerto delle dame principalissime di Margherita Gonzaga d'Este. Firenze: SPES.

Durante, E., \& Martellotti A. (2010). 'Giovinetta peregrina'. La vera storia di Laura Peperara e Torquato Tasso. Firenze: Olschki.

Enciclopedia del Diritto (1958-1981). Milano: Giuffrè.

Fabbri, P. (1985). Monteverdi. Torino: EdT.

Ferrone, S., Burattelli, C., Landolfi, D., \& Zinanni, A. (Eds). (1993). Comici dell'Arte. Corrispondenze: G. B. Andreini, N. Barbieri, P. M. Cecchini, S. Fiorillo, T. Martinelli, F. Scala (2 voll). Firenze: Le Lettere.

Ferrone, S. (1993). Attori mercanti corsari. Torino: Einaudi.

Follino, F. (1608). Compendio delle sontuose feste fatte l'anno MDCVIII nella Città di Mantova, per le Reali Nozze del Serenissimo Prencipe D. Francesco Gonzaga, con la Serenissima Infante Margherita di Savoia. Mantova: Aurelio \& Lodovico Osanna.

Gallico, C. (Ed.). (2004). Federico Follino. Cronache mantovane (1587$-1608)$. Firenze: Olschki.

Harrán, D. (1987). Salomone Rossi As a Composer of Theater Music. Studi Musicali, 16, 96-131.

Harrán, D. (Ed.). (1995). Salamone Rossi: complete works. Neuhausen: Hänssler-Verlag.

Kirkendale W. (1993). The Court Musicians in Florence During the Principate of the Medici. Firenze: Olschki.

Megale, T. (2016). Partenope sul Mincio. Relazioni teatrali tra Napoli e Mantova in età moderna. In S. Brunetti (Ed.), Maestranze, artisti e apparatori per la scena dei Gonzaga 1480-1630 (pp. 405-421). Bari: Edizioni di pagina.

Monaldini, S. (2000). L'Orto dell'Esperidi. Musici. Attori e artisti nel patrocinio della famiglia Bentivoglio (1646-1685). Lucca: LIM.

Musiche de alcuni eccellentissimi musici composte per La Maddalena, sacra rappresentazione di Gio. Battista Andreini fiorentino (1617). Venezia: Stampa del Gardano presso Bartolomeo Magni.

Neue Deutsche Biographie (1953-). Berlin: Dunker \& Humblot.

Parisi, S.H. (1989). Ducal patronage of music in Mantua, 1587-1627: an archival study (Ph.D. Thesis). University of Illinois at Urbana-Champaign: UMI Research Press.

Pertile, P. (1894). Storia del diritto italiano. Torino: UTET. 
Portioli, A. (1882). Il matrimonio di Ferdinando Gonzaga con Caterina de' Medici. Mantova: Mondovi.

Rollandi, M.S., \& Romani M. (2018). Tesori ovvero beni denaro-equivalenti. Considerazioni sulle funzioni degli oggetti nell'antico regime (secoli XV-XVIII). Società e storia, 159, 1-34.

Solerti, A. (1903). Gli albori del melodramma. Torino: Bocca.

Solerti, A. (1905). Musica, ballo e drammatica alla Corte Medicea dal 1600 al 1637. Firenze: Bemporad.

Weaver, R.L., \& Wright Weaver, N. (1978). A Cronology of Music in the Florentine Theater. Detroit: Information Coordinators, Inc.

Riassunto: Nell'archivio di stato di Mantova sono conservati numerosi atti notarili, che coinvolgono alcuni fra i più famosi comici del Seicento. Piermaria Cecchini (Frittellino), Francesco Andreini e i suoi figli Domenico e Giacinto, Giovan Battista (Lelio) con suo figlio Pietro Enrico, Tristano Martinelli e altri, ricorsero ai notai per i loro testamenti, contratti d'acquisto e vendita, liberazione da crediti, inventari di beni e doti. Alcuni di quegli atti interessano anche i membri femminili della famiglia Andreini: Lavinia (suor Fulvia), Caterina (forse suor Clarastella), Virginia Ramponi Andreini (Florinda). In particolare, un atto di emancipazione (1620) toccò indirettamente Virginia (Genova, 1583 - ?, ante 17 novembre 1631), prima moglie di Giovan Battista Andreini. Sulla base dell'analisi del documento, vengono trattate due prospettive: il peculiare contributo di Virginia alle attività della famiglia anche sotto il profilo economico; lo stato delle donne nei confronti dell'emancipazione, intesa sotto il profilo giuridico che era patriarcale e difensivo dei patrimoni. Il documento diviene quindi occasione per riflettere, partendo da Virginia ma spingendo poi lo sguardo oltre, sulla condizione delle donne attive nel mondo teatrale e musicale fra giurisprudenza, professioni artistiche, patrimoni economici e vita quotidiana. In conclusione, attraverso alcuni selezionati esempi (il concerto delle dame di Ferrara, Adriana Basile, Margherita Salicola, Antonia Merighi), il tema dell'emancipazione viene osservato in tutto il Seicento, quando le cantanti donne popolavano il nuovo mercato operistico con successo, anche economico. Poiché la legge era rimasta invariata, esse sperimentarono diverse strategie per tutelare la propria persona e i propri patrimoni.

Parole chiave: attrici, cantanti donne, opera, emancipazione, diritto 